\title{
Multifrequency interferometer and radio continuum monitoring observations of CTA $102^{\star}$
}

\author{
F. T. Rantakyrö ${ }^{1,2, \star \star}$, K. Wiik ${ }^{3}$, M. Tornikoski ${ }^{4}$, E. Valtaoja ${ }^{3,5}$, and L. B. Bååth ${ }^{6}$ \\ ${ }^{1}$ European Southern Observatory, Casilla 19001, Santiago 19, Chile \\ 2 Observatorio Cerro Calan, Universidad de Chile, Santiago, Chile \\ 3 Tuorla Observatory, Väisäläntie 20, 21500 Piikkiö, Finland \\ ${ }^{4}$ Metsähovi Radio Observatory, Metsähovintie 114, 02540 Kylmälä, Finland \\ 5 Department of Physical Sciences, University of Turku, 20100 Turku, Finland \\ ${ }^{6}$ Center for Imaging Technologies, Halmstad University, 30118 Halmstad, Sweden
}

Received 12 March 2002 / Accepted 31 March 2003

\begin{abstract}
This paper represents the work of several years of observation of CTA 102 with both single dish telescopes and interferometric arrays, covering wavelengths from $1.2 \mathrm{~cm}$ to $1.3 \mathrm{~mm}$. The resolution of the maps covers an order of magnitude from 0.5 mas to $50 \mu$ as. The images presented demonstrate the rapid structural changes in this source. These rapid structural changes correlates with the outbursts seen in the total flux, turnover frequency and in the spectral index. We have calculated the Brightness Temperatures of the components $\left(T_{\mathrm{B}}\right)$ directly from the determined component sizes and also using the logarithmic variability amplitudes and timescales to calculate the $T_{\mathrm{B}} \mathrm{s}$. Both the methods yielded measurements of source frame $T_{B} \mathrm{~s}$ no higher than $\sim$ a few $\times 10^{12} \mathrm{~K}$. We find that the observed average proper motion is $\sim 0.4 \pm 0.07 \mathrm{mas} / \mathrm{yr}$, which corresponds to an apparent transverse velocity of $11 \pm 2 \mathrm{c}$.
\end{abstract}

Key words. shock waves - quasars: individual CTA102 - galaxies: jets - radiation mechanisms: non-thermal

\section{Introduction}

CTA 102 (QSO 2230+114, $\alpha=223236.41$ and $\delta=$ +114350.9 (J2000), $z=1.037$, and $m_{v}=17.33(\mathrm{NED}$ database)) has been studied at several wavelengths and during many epochs. It has been classified as an optical variable with a mean magnitude of 17.33 and as a High Polarized Quasar (HPQ). It was the first radio source for which variations in the flux density were reported (Scholomitski 1965; Dent 1965). The time-scale of the variations led Sholomitski to deduce a linear size of $0.1 \mathrm{pc}$ for the radio emitting region. An angular size of 10 milliarcsec (mas) was deduced by Slish (1963) from the spectral turnover, but at the red shift distance of CTA 102 this implies that the region responsible for the synchrotron emission is larger than the variability timescale suggests. With the inferred angular size the brightness temperature of the variable component would exceed the inverse Compton limit and a very large X-ray flux density should be observed. The dilemma of "Superluminal Flux Variations"

Send offprint requests to: F. T. Rantakyrö,

e-mail: frantaky@eso.org

* Based on observations collected at the European Southern Observatory, Chile.

$\star \star$ Present address: European Southern Observatory, Casilla 19001, Santiago 19, Chile.
(Romney et al. 1984) can be solved by either an intrinsic model based on bulk relativistic motion of the radio emitting material (Blandford \& Königl 1979; Scheuer \& Readhead 1979) or an extrinsic model based on refractive interstellar scintillation (Rickett et al. 1984; Rickett 1986). Rantakyrö et al. (1996) showed that the major contribution to the variability at wavelengths shorter than $\lambda 32 \mathrm{~cm}$ is due to intrinsic processes.

Observations of CTA 102 with the EGRET telescope at the high-energy $\gamma$-rays $(>100 \mathrm{MeV})$ have shown that the source exhibits a high $\gamma$-ray luminosity, $L_{\gamma}=5 \times 10^{47} \mathrm{erg} \mathrm{s}^{-1}$ (Nolan et al. 1993), and the X-ray flux is correspondingly high, $F_{X}=$ $0.75 \mu \mathrm{Jy} . L_{\gamma}$ dominates the emission seen at all other wavelengths, a common feature among gamma-bright blazars. The common explanation for the high apparent $L_{\gamma}$ is that the emission is the result of a beamed jet with a high Lorenz factor.

The arc second scale structure of the source is dominated by a central core and two other components (Spencer et al. 1989). At $\lambda 18 \mathrm{~cm}$ the stronger component has a flux density of $0.2 \mathrm{Jy}$ and is located at $\sim 1.6 \operatorname{arcsec}$ at $\mathrm{PA} \sim 140^{\circ}$, the fainter one is only $0.1 \mathrm{Jy}$ at 1.0 arcsec at PA $\sim-40^{\circ}$. Both components have a steep spectrum. Observations at $\lambda 6 \mathrm{~cm}$ (Wehrle \& Cohen 1989), at $\lambda 18 \mathrm{~cm}$, and at $\lambda 1.3 \mathrm{~cm}$ (Rantakyrö et al. 1996) show a central double knot feature (separated in NS direction by $\sim 3$ mas) with an extended diffuse tail bending sharply to the SW. Rantakyrö et al. (1996) showed that the source exhibits 
very fast structural changes. Due to this rapidity together with the long time between the epochs the proper motion was underestimated to be only $\mu=0 \pm 0.5$ mas/year.

Typical for the major outbursts we observe in blazar and gamma-bright AGNs is that they first start with a rapid flux increase simultaneously at optical and IR frequencies and later at the high radio frequencies $(86 \mathrm{GHz}$ and higher) (Courvoisier et al. 1988). High frequency single dish monitoring shows that outbursts tend to emerge almost simultaneously over the range $86-300 \mathrm{GHz}$ with the turnover frequency reaching $86 \mathrm{GHz}$ within a month and then $22 \mathrm{GHz}$ within 4-5 months (Lainela et al. 1993; Stevens et al. 1996). Similar time-scales are observed in 3C 279 (Litchfield et al. 1995). This has been explained (Marscher \& Gear 1985; Marscher et al. 1992) by a thin shock which is formed close to the central engine and then moves down the jet. After some time the shock will expand adiabatically and the spectral turnover (due to synchrotron selfabsorption) will move towards lower frequencies. The outburst will therefore be observed at lower frequencies after the expansion has started. Observations of the shocks at their early stages of development are fundamental to our understanding of how they are formed, how they emerge from the core, and how they develop on their way through the radio jet. It must be emphasized that such observations can only be done with very high angular resolution (to resolve the features) and at high frequencies (as the region close to the core is optically thick at low frequencies) thus mmVLBI is the only way to image the shocks as they emerge from the core.

The April 1990 session was the first epoch when we could produce hybrid maps using intercontinental baselines to obtain a resolution of $50 \mu$ as (Rantakyrö et al. 1998). This was achieved because of the development of new receivers, improved phase stability of local oscillators, new data reduction techniques (Bååth et al. 1992), and the increase in the source flux density.

Our earlier observations (Bååth et al. 1992; Rantakyrö et al. 1998) have shown that the radio cores of powerful AGNs (in the observer's rest frame) are very small, on the order of $10^{16}-10^{18} \mathrm{~cm}$, which is only 5-5000 times larger than the Schwarzschild radius of a $10^{9} M_{\odot}$ black hole.

A special and characteristic feature of the submilliarcsecond scale structures is that the curvature observed with $\mathrm{cm}$-VLBI seems to continue, but is further enhanced, closer to the core (Rantakyrö et al. 1998).

This paper presents four epochs of the Coordinated mmVLBI Array (CMVA) $\lambda 3 \mathrm{~mm}$ VLBI, $\lambda 7 \mathrm{~mm}$ and $\lambda 13 \mathrm{~mm}$ dualfrequency VLBA observations, three epochs of global $22 \mathrm{GHz}$ VLBI observations, one adhoc EVN $22 \mathrm{GHz}$ epoch, and continuum monitoring of this source at 22 [13 mm], 37 [8 mm], $90[3 \mathrm{~mm}]$, and $230 \mathrm{GHz}[1.3 \mathrm{~mm}]$. We will also discuss and analyse these observations.

\section{Observations and data reduction}

\section{1. $3 \mathrm{~mm} \mathrm{VLBI}$}

The CMVA 1997 sessions were global $86 \mathrm{GHz} \lambda 3 \mathrm{~mm}-$ VLBI sessions involving the following telescopes: Effelsberg
Table 1. CMVA Telescope information. $D$ is the diameter in meters, $\mu$ is the sensitivity in $\mathrm{K} / \mathrm{Jy}, T_{\text {sys }}$ the single-sideband system equivalent noise in Kelvin referred to outside the atmosphere, and the final column gives information about the sessions (April/Jun./Oct./Dec.) in which the telescope participated.

\begin{tabular}{lllll}
\hline \hline Name & $\begin{array}{l}\text { D } \\
{[\mathrm{m}]}\end{array}$ & $\begin{array}{l}\mu \\
{[\mathrm{Jy} / \mathrm{K}]}\end{array}$ & $\begin{array}{l}T_{\text {sys }} \\
{[\mathrm{K}]}\end{array}$ & Sessions \\
\hline Effelsberg & 100 & 7.7 & 800 & yes/no/yes/no \\
Hat Creek & $3 \times 6.1$ & 90 & 420 & yes/no/no/yes \\
Haystack & 37 & 17 & 400 & yes/yes/yes/yes \\
Kitt Peak & 12 & 53 & 205 & yes/yes/yes/yes \\
Metsähovi & 14 & 58 & $300-$ & no/no/yes/no \\
Onsala & 20 & 35 & 480 & yes/yes/yes/yes \\
Owens Valley & 10.4 & 46 & 500 & yes/yes/no/no \\
Pico Veleta & 30 & 7.1 & 200 & yes/no/yes/no \\
Pie Town & & & & yes/yes/yes/yes \\
Quabbin & 14 & 42 & 320 & yes/no/no/yes \\
\hline
\end{tabular}

(Germany), Hat Creek (USA), Haystack (USA), Kitt Peak (USA), Metsähovi (Finland), Onsala (Sweden), Owens Valley (USA), Pico Veleta (Spain), Pie Town (USA) and Quabbin (USA). Information on the participating antennas can be found in Table 1. Especially the June 1997 epoch suffered from the small number of detected fringes and participating telescopes. The data were recorded using MarkIII (and compatible) recorders to thick tapes and using $14 \mathrm{IF}$-channels with a $4 \mathrm{MHz}$ bandwidth in each, and later correlated at the Haystack correlator. The output from the correlator was read into the Haystack Observatory Post-processing System (HOPS) post-correlation package and the fringe search and amplitude calibration was done within this package.

\subsection{EVN, Global VLBI, and VLBA observations}

The VLBI sessions (November 13th 1992; September 20th 1993; October 19th 1996) were global sessions including the VLBA, VLA, and other available telescopes. The VLBA session (June 24th 1998) was a dual-frequency $22 / 43 \mathrm{GHz}$ observation. In March 1994 we did a short adhoc observation using the EVN antennas. Being an adhoc observation the quality of the image is much less than what is expected from a full experiment. Table 2 lists the antennas participating in these sessions. The participating antennas are Medicina (Italy), Noto (Italy), Metsähovi (Finland), Onsala (Sweden), NRAO 140 (Green Bank, USA), Effelsberg (Germany), Jodrell Bank Mk2 (England), VLBA $\mathrm{BR}_{\text {(Brewster), VLBA }}$ (Fo (Fort Davis), VLBA $_{K P}$ (Kitt Peak), VLBA LA $_{\text {(Los Alamos), VLBA }}$ (Pie Town), VLBA ${ }_{\mathrm{OV}}$ (Owens Valley), VLBA $\mathrm{NL}$ (North Liberty), VLBA $_{S C}$ (Saint Croix), VLBA ${ }_{M K}$ (Mauna Kea) and VLBA (Hancock) and one antenna from the Very Large Array (VLA).

The data were recorded using MarkIII and VLBA recorders to thick tapes, and using $14 \mathrm{IF}-$ channels with a $4 \mathrm{MHz}$ bandwidth in each, and later correlated at the VLBA correlator. The output from the correlator was read into the AIPS package and the fringe search and amplitude calibration was done within this package. The amplitude calibration was done by applying the measured system temperature at each station, correcting for 
Table 2. EVN, Global and VLBA Telescope participation information. The participation of each telescope is listed by epoch $(1992 / 1993 / 1994 / 1996 / 1998)$.

\begin{tabular}{lrrrrr}
\hline \hline Name & 1992 & 1993 & 1994 & 1996 & 1998 \\
& & & & & \\
\hline Medicina & yes & yes & yes & yes & no \\
Noto & yes & yes & yes & yes & no \\
Metsähovi & yes & yes & yes & yes & no \\
Onsala & yes & yes & yes & yes & no \\
NRAO 140 & yes & yes & no & yes & no \\
Effelsberg & yes & yes & yes & yes & no \\
Jodrell Bank & no & yes & yes & yes & no \\
VLBA $_{\text {BR }}$ & yes & yes & no & yes & yes \\
VLBA $_{F D}$ & no & yes & no & yes & yes \\
VLBA $_{\text {LA }}$ & yes & yes & no & yes & yes \\
VLBA $_{P T}$ & yes & yes & no & yes & yes \\
VLBA $_{\mathrm{OV}}$ & yes & yes & no & yes & yes \\
VLBA $_{\mathrm{NL}}$ & yes & yes & no & yes & yes \\
VLBA $_{S C}$ & no & yes & no & yes & yes \\
VLBA $_{\mathrm{MK}}$ & no & yes & no & yes & yes \\
VLBA $_{\mathrm{KP}}$ & yes & yes & no & yes & yes \\
VLBA $_{\mathrm{HN}}$ & yes & no & no & no & yes \\
VLA (USA) $^{\text {no }}$ & yes & no & yes & no \\
\hline
\end{tabular}

gain and atmospheric effects. We believe that the amplitudes are correct to within $10 \%$.

The later uv data model fitting was done in the DIFMAP package (Shepherd 1997). We first fitted Gaussian components directly to the uv-data and the final images were constructed by adding components using the differential mapping approach.

\subsection{Continuum monitoring}

CTA 102 was observed at $\lambda 3 \mathrm{~mm}[90 \mathrm{GHz}]$ and $\lambda 1.3 \mathrm{~mm}[230 \mathrm{GHz}]$ with the SEST at the European Southern Observatory site on Cerro La Silla in Chile (Fig. 2). Some of the data have been published in Tornikoski et al. (1996). At $\lambda 8 \mathrm{~mm}[37 \mathrm{GHz}]$ and $\lambda 13 \mathrm{~mm}[22 \mathrm{GHz}]$ it was monitored by the Metsähovi group as part of a larger sample of AGNs (Teräsranta et al. 1998) using the Metsähovi Radio Telescope (Fig. 1). The SEST observations at $90 \mathrm{GHz}$ were made using a dual-polarization Schottky receiver until June 1995, and since then using a SIS receiver. As a backend, a wide band $(1 \mathrm{GHz})$ AOS was used. For the $230 \mathrm{GHz}$ observations a Schottky receiver and a wide band AOS were initially used, but since 1991 the data were mainly obtained with a single channel bolometer. Some of the recent data points were obtained with a SIS+AOS setup. At Metsähovi the 22 and $37 \mathrm{GHz}$ observations were made using a HEMT frontend. At the SEST the flux density observations were made with dual-beamswitching mode with either 10 or $20 \mathrm{~s}$ spectra. The mean and RMS of the observation was calculated by doing a least-squares fit to the flux and RMS obtained from each individual spectra. There were typically 20 spectra in each observation run. The calibration of the flux densities used the standard SEST calibration based on measurement of planet fluxes. At Metsähovi the continuum receivers at 22 and $37 \mathrm{GHz}$ were used with $20 \mathrm{~s}$ scans with a total integration time

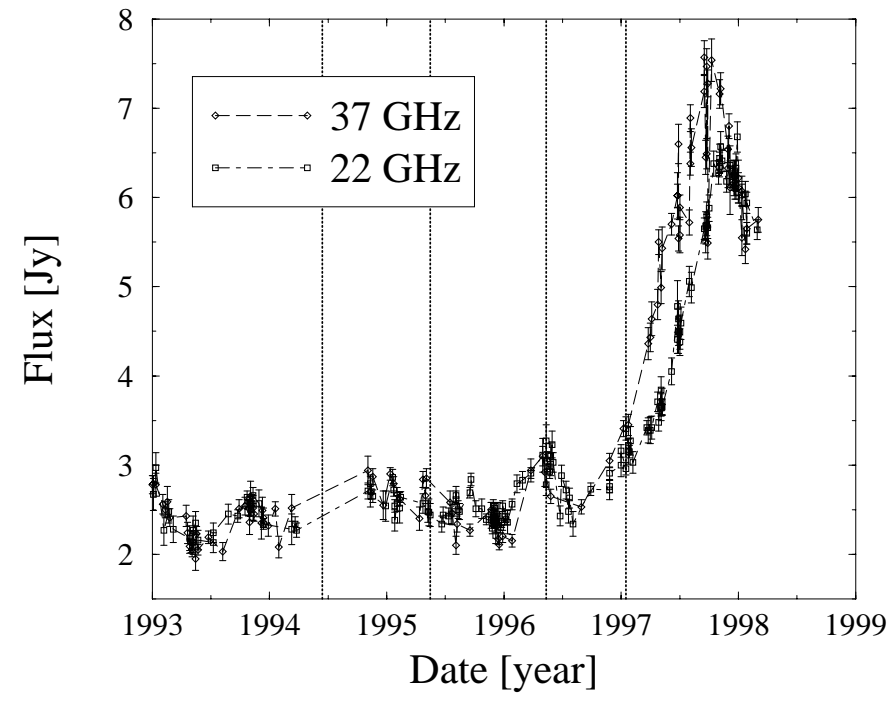

Fig. 1. 22 and $37 \mathrm{GHz}$ continuum observations of CTA 102. Diamonds markers with the long dashed line are the $22 \mathrm{GHz}$ observations and the squares with the short dash long dash line represents the $37 \mathrm{GHz}$ observations. The vertical dotted lines indicate the estimated dates for the zero epochs from the proper motion estimates (see Sect. 4). The error bars indicate the total error of the measurement which consists of errors in calibration, pointing, and the the thermal noise of the measurement.

of $1200 \mathrm{~s}$ for each observation. See Teräsranta et al. (1998) for an extensive description of the calibration method used in these observations. The result of this monitoring is presented in Figs. 1-2. It is evident that the outbursts are first seen at 90 and $230 \mathrm{GHz}$ (the poor time sampling of the $230 \mathrm{GHz}$ precludes the determination of the time-delay between these two frequencies) and later appear simultaneously at 22 and $37 \mathrm{GHz}$. This agrees very well with what has been seen previously (Lainela et al. 1993; Stevens et al. 1996; Litchfield et al. 1995). Unfortunately the flux outburst seen at 22 and $37 \mathrm{GHz}$ in early 1996 occurs at the time where we have no higher frequency measurements. The multifrequency variability behavior of CTA102 will be discussed in more detail in Tornikoski et al. (2002).

\section{Observations}

\section{1. $\lambda 3 \mathrm{~mm}-\mathrm{VLB}$ observations with the CMVA in 1997}

The $\lambda 3 \mathrm{~mm}$-VLBI observations with the CMVA in 1997 are very strongly limited by the small number of detected fringes. Thus we present a more extensive discussion of the uv-data for these epochs. Shown in Fig. 3 are the amplitudes with baseline lengths for each epoch and similarly in Fig. 4 we show the uv-coverage of the observations in 1997. The most critical shortcoming of the observations is the low number of uv-data points. By using HOPS with incoherent averaging we obtained one data point for each baseline and $6.5 \mathrm{~min}$ scan. Thus each data point is highly significant and we have been very conservative in our editing (i.e. removal of data points).

Subsequent uv data model fitting was done using the DIFMAP package. We fitted elliptical Gaussian components 


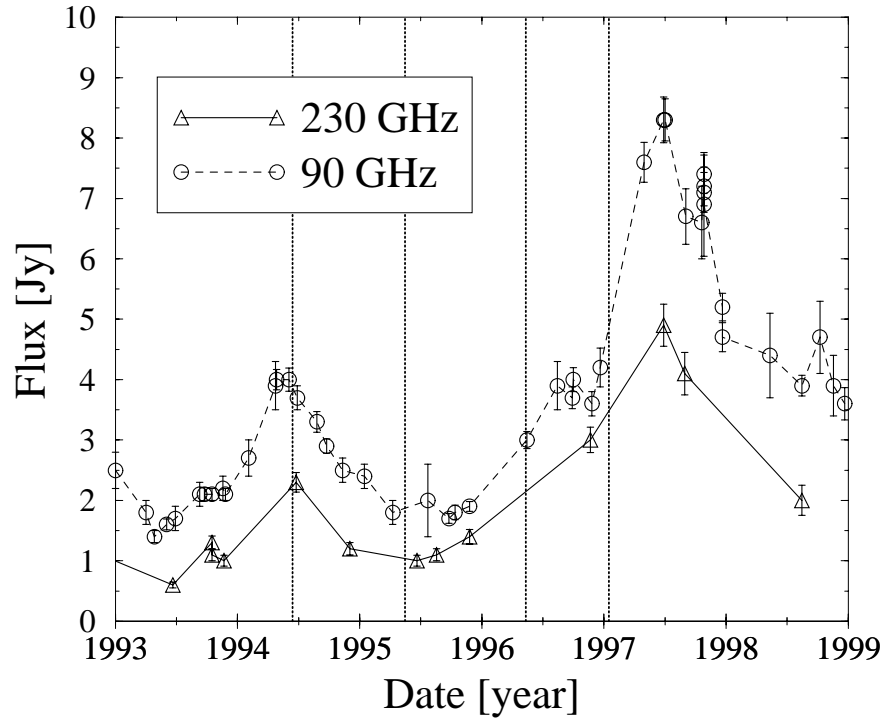

Fig. 2. 90 and $230 \mathrm{GHz}$ continuum observations of CTA 102. Circles with the dashed line are the $3 \mathrm{~mm}$ observations and the triangles with the continuous line represents the $1.3 \mathrm{~mm}$ observations. The vertical dotted lines indicate the estimated dates for the zero epochs from the proper motion estimates (see Sect. 4). The error bars indicate the errors in the measurements and are the result of the measurement RMS errors and the calibration and pointing errors.

directly to the uv-data, until adding components to the fit did not significantly decrease the $\chi^{2}$ value and a good fit to the closure phases was achieved. The final images were constructed using these model fits and hybrid mapping. The final uv-coverage varied so much between the epochs that no common restoring beam could be used (see the uv-coverages in Fig. 4). The final images of the four epochs are shown in Fig. 5 and the results from the $\chi^{2}$-fit are listed in Table 3 . The errors in the parameters are typically in the order of $<15 \%$. This error estimate is based on the spread of the fits yielding similar $\chi^{2}$ values.

Note: in the following discussion it is important to remember that we are not discussing the images but components of the images. The specific size, structure, and location of each component may be open for discussion, but the existence, general position, and rough size, of the components are defined and do not vary significantly with the number of components used in the fit or the actual model fitting method used (using the uvdata model-fitting routine in DIFMAP or in AIPS).

\section{2. $22 \mathrm{GHz}$ global and EVN VLBI observations}

These observations were part of the projects Global VLBI (GL010, GL013, GL014, and GW014 (Wiik et al. 2001), and the adhoc EVN project GR005. The global VLBI projects consisted of snapshot observations of several QSOs, thus the restoring beam and signal to noise ratio are worse than expected for a full run. Thus although the 3 epochs have similar antenna configuration, the final restoring beam and SNR vary. We present the three images in Fig. 6.

The EVN observation was made with the antennas that were $22 \mathrm{GHz}$-capable at that time. With the limited
Table 3. Model parameters for the elliptical Gaussian components fitted to the CTA $102 \lambda 3 \mathrm{~mm}$-VLBI maps made in the four CMVA epochs in 1997. Date is the epoch of observation, ID is the label identifying the component, $\Delta \alpha$ and $\Delta \delta$ give the position of the component relative to the core, Flux is the flux density of the component, $b_{\text {maj }}$ and $b_{\min }$ are the major and minor axes of the elliptical Gaussians $(F W H M)$, and PA is the position angle of the fitted Gaussians.

\begin{tabular}{lcrrrrrr}
\hline \hline Date & ID & $\begin{array}{r}\Delta \alpha \\
{[\mathrm{mas}]}\end{array}$ & $\begin{array}{r}\Delta \delta \\
{[\mathrm{mas}]}\end{array}$ & $\begin{array}{r}\text { Flux } \\
{[\mathrm{Jy}]}\end{array}$ & $\begin{array}{r}b_{\text {maj }} \\
{[\mathrm{mas}]}\end{array}$ & $\begin{array}{r}b_{\min } \\
{[\mathrm{mas}]}\end{array}$ & $\begin{array}{r}\text { PA } \\
{\left[{ }^{\circ}\right]}\end{array}$ \\
\hline Apr. & I0 & 0 & 0 & 2.55 & 0.05 & $<0.001$ & 60 \\
Apr. & I1 & 0.06 & -0.12 & 0.01 & 0.14 & $<0.001$ & -16 \\
Apr. & I2 & 0.61 & -0.16 & 0.13 & 0.2 & $<0.001$ & 22 \\
Apr. & I3 & 0.54 & -0.59 & 0.06 & 0.2 & $<0.001$ & -17 \\
Jun. & J0 & 0 & 0 & 1.48 & 0.4 & 0.025 & -4 \\
Jun. & J1 & 0.20 & 0.01 & 4.1 & 1.0 & $<0.001$ & -22 \\
Jun. & J2 & 0.62 & -1.20 & 1.96 & 0.8 & $<0.001$ & 70 \\
Oct. & K0 & 0 & 0 & 0.35 & 0.3 & $<0.001$ & 77 \\
Oct. & K1 & 0.11 & -0.38 & 0.77 & 0.2 & $<0.001$ & 60 \\
Oct. & K2 & 0.33 & -0.32 & 1.1 & 0.14 & $<0.001$ & 50 \\
Oct. & K3 & 0.49 & -0.68 & 0.74 & 0.26 & $<0.001$ & 70 \\
Oct. & K4 & 1.84 & -1.2 & 0.3 & 0.2 & $<0.001$ & 50 \\
Oct. & K5 & 1.84 & -1.9 & 0.6 & 0.4 & $<0.001$ & 60 \\
Dec. & L0 & 0 & 0 & 1.0 & 0.1 & $<0.001$ & -35 \\
Dec. & L1 & 0.09 & -0.39 & 0.54 & 0.08 & 0.05 & -30 \\
Dec. & L2 & 0.37 & -0.70 & 0.14 & 0.1 & $<0.001$ & 0 \\
Dec. & L3 & 0.66 & -0.66 & 0.10 & 0.2 & $<0.001$ & -16 \\
Dec. & L4 & 0.90 & -0.76 & 0.12 & 0.3 & $<0.001$ & -22 \\
\hline
\end{tabular}

uv-coverage and the short observing time, the final image has much poorer resolution and SNR than what is currently available with the EVN. The final hybrid mapping image is shown in Fig. 7. The error in the absolute calibration is much worse, estimated to be $\sim 40 \%$. This map is presented here as it gives us crucial information about the structural changes in the source.

To obtain the position of the underlying components we have fitted Gaussian components to the uv-data. Due to the complexity of the image as many as 9 Gaussians have been needed to find a good fit to the data. The number of required components was estimated by adding components until the fit to the closure phases did not improve. An additional criterion was that the $\chi^{2}$ should decrease significantly with an added component. The component positions, sizes, and fluxes for the three epochs are presented in Table 4 . To test the convergence, we used several sets of starting values for the iterative solution, and the values shown in Table 4 were the best possible ones (minimum $\chi^{2}$ was reached). The errors in these solutions are typically in the order of $<15 \%$. This number is based on the spread of fits yielding similar $\chi^{2}$ numbers. The components have been labeled so that they are uniquely identified. We will discuss the intra epoch identification of components and their apparent proper motion in Sect. 4.

\subsection{3 and $22 \mathrm{GHz}$ VLBA observations June 24th 1998}

In Fig. 8 we present the complete maps from the dualfrequency (22 and $43 \mathrm{GHz}$ ) VLBA observations made in June 24th 1998. There is no structure above the noise level 

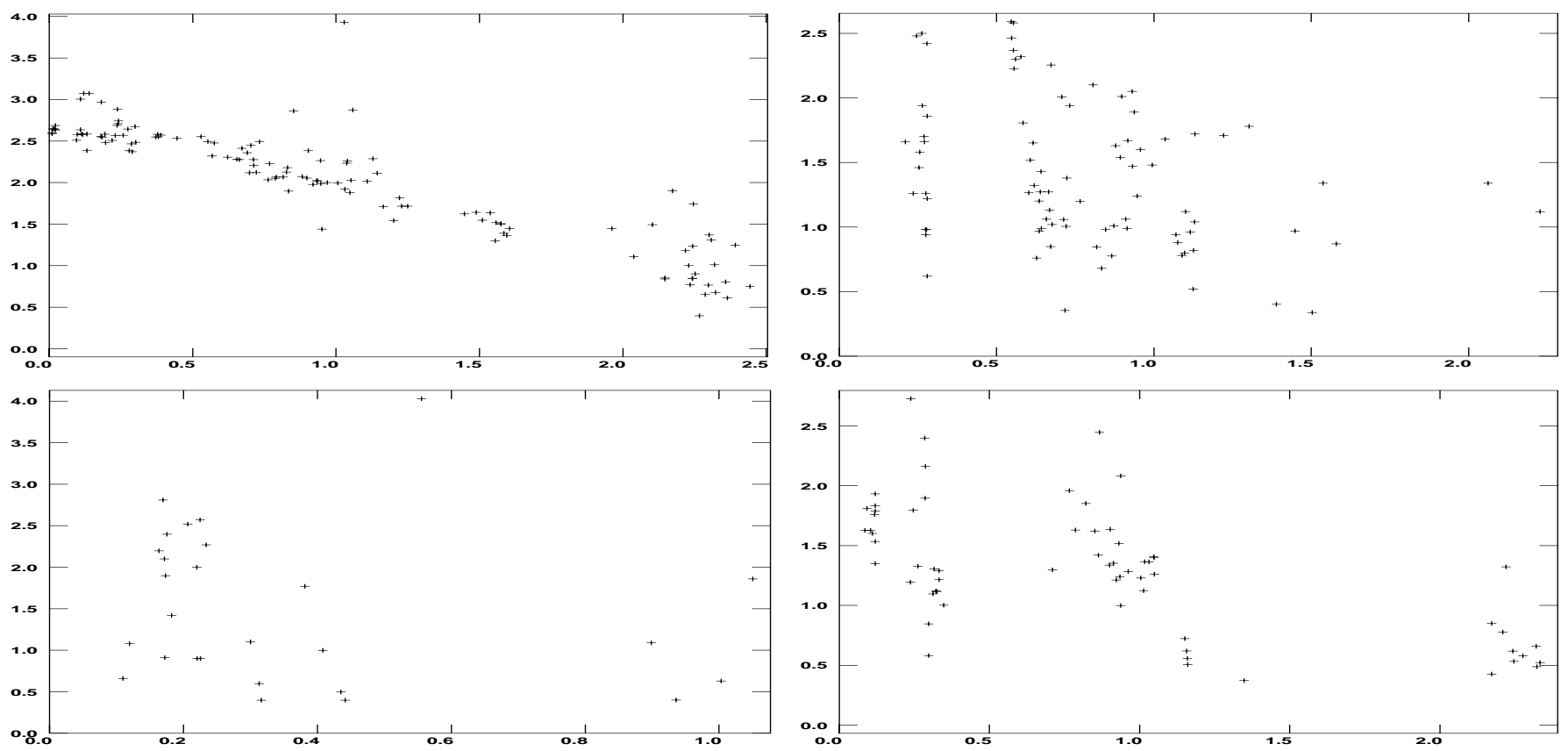

Fig. 3. Plots of uv amplitude vs. baseline length for the four epochs in 1997 with the CMVA. The values given on the $x$-axis are in G $\lambda$ and on the $y$-axis in Janskys in all four plots. Top left: April 14, top right: June 18, bottom left: October 24, and bottom right: December 13.
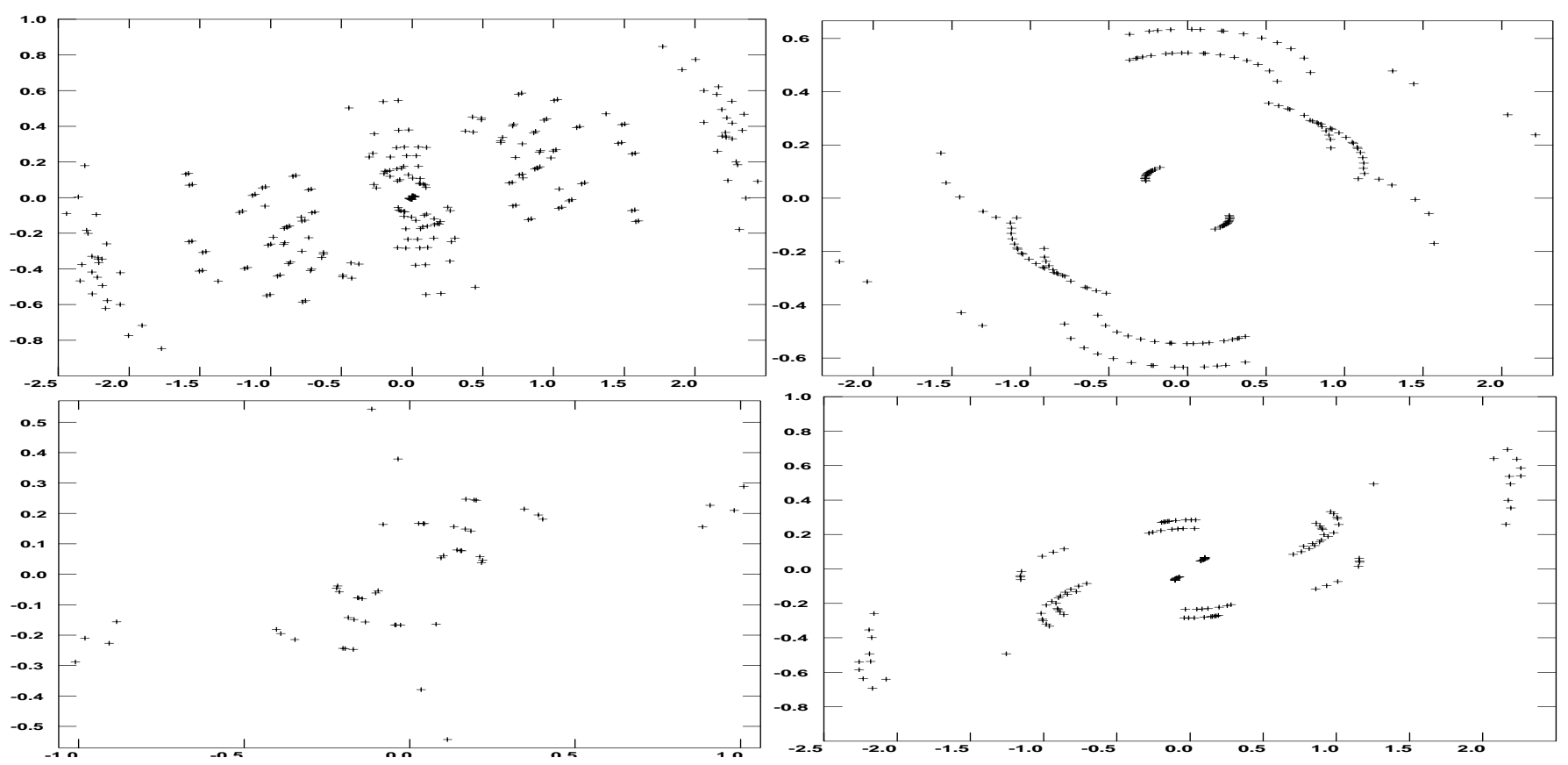

Fig. 4. CTA 102 Plots of uv-coverage for the four epochs with the CMVA in 1997. The values on both axes are in G $\lambda$ in all four plots. Top left: April 14, top right: June 18, bottom left: October 24, and bottom right: December 13.

outside the region shown in the two maps. The resulting modelfit of elliptical Gaussians to the calibrated uv-data is shown in Table 5. The discussion about the validity and fidelity of the $\chi^{2}$-fits made in Sect. 3.2 is also valid for these fits.

\subsection{Spectral index}

The spectral index is defined as $S \propto v^{\alpha}$, and thus we can calculate $\alpha$ from our flux monitoring measurements (Figs. 1-2) taken at the SEST and Metsähovi. The result is presented in
Figs. 9-10. Included in the figures are also the estimated dates for the zero epochs from the proper motion estimates (see Sect. 4).

The quiescent spectra, as defined by the minimum fluxes, from $1995.0 / 1995.5 \quad(37-90 \mathrm{GHz} / 22-37 \mathrm{GHz})$ to $1996.4 / 1996.4$ are typical falling spectra, but the flaring spectrum is highly inverted. At all times the turnover frequency is higher than $37 \mathrm{GHz}$ except during late 1995 and early 1996, and for a substantial fraction of the time in conjunction with the flux outbursts higher than $90 \mathrm{GHz}$. 

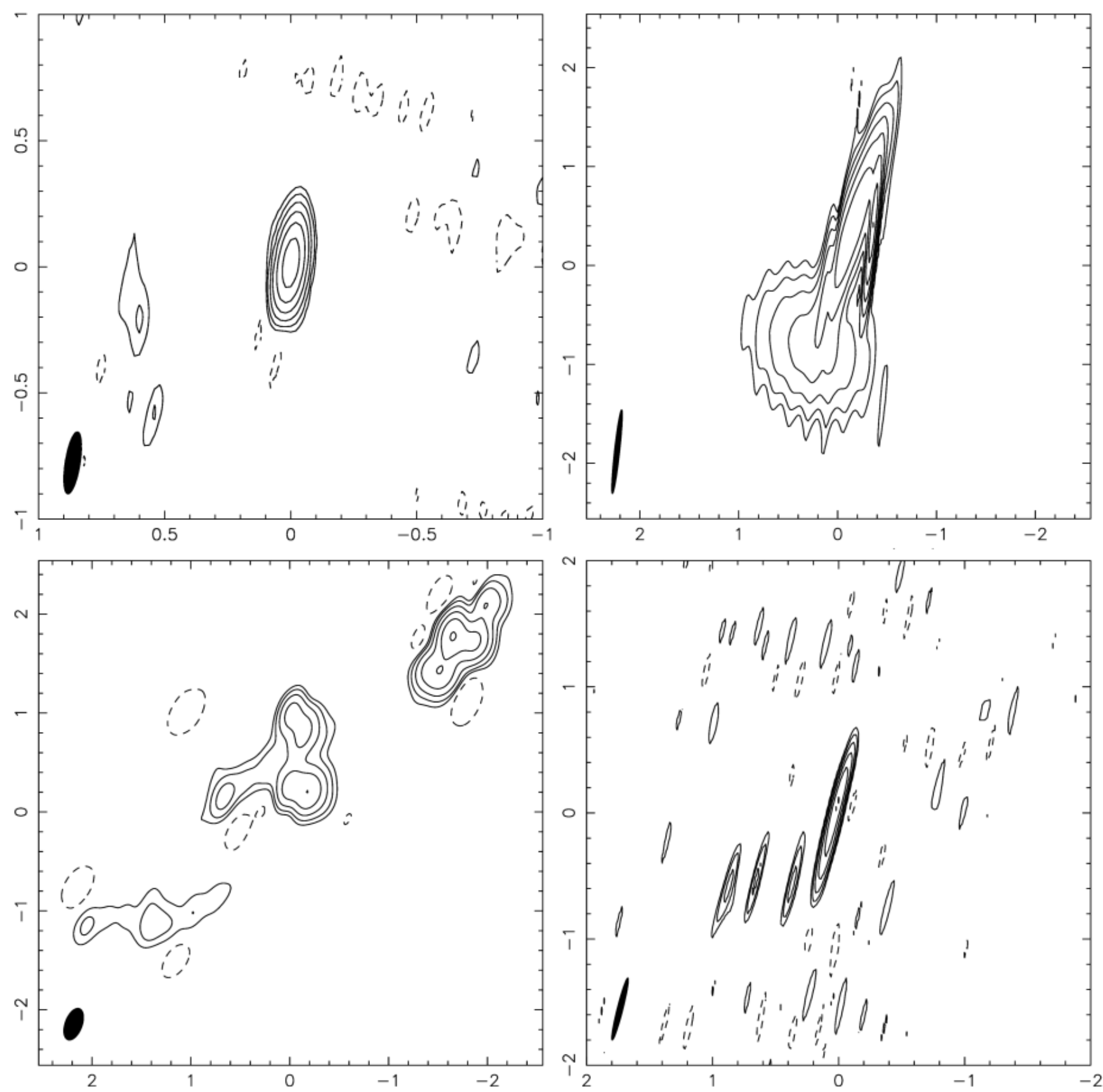

Fig. 5. CTA $10286 \mathrm{GHz}$ CMVA images from 1997. The map scales are all in mas. Top left: April 14, Peak flux 1.86 Jy/beam, and restoring beam $0.25 \times 0.06$ mas at $-9^{\circ}$. Contour levels are $-2,2,4,8,16,32,64 \% \times$ Peak flux. Top right: June 18 , Peak flux $1.24 \mathrm{Jy} / \mathrm{beam}$, and restoring beam $0.85 \times 0.05$ mas at $-6^{\circ}$. Contour levels are $-1,1,2,4,8,16,32,64 \% \times$ Peak flux. Bottom Left: October 24, Peak flux $0.89 \mathrm{Jy} /$ beam, and restoring beam $0.33 \times 0.17$ mas at $-20^{\circ}$. Contour levels are $-3,3,6,12,24,48,96 \% \times$ Peak flux. Bottom right: December 13 , Peak flux $0.81 \mathrm{Jy} /$ beam, and restoring beam $0.5 \times 0.04$ mas at $-14^{\circ}$. Contour levels are the same as in October 24 .

The spectral index turnover frequency is at all times lower than $230 \mathrm{GHz}$. Note that the sparse sampling at 90 and 230 precludes any detailed analysis of the short time-scale evolution of the spectral index as well as an accurate determination of the time delay between 90 and $230 \mathrm{GHz}$.

It is evident from the total flux monitoring (Figs. 1-2) and the estimates of the zero epochs (Table 6) that at the start (or with a delay of some weeks) of each large outburst there is an ejection of a component from the core. Thus the total flux monitoring is a valuable tool in helping us to understand the structural changes in this source. This is also seen in the spectral index plots (Figs. 9-10) where each ejection causes a steepening in the spectrum. Better timesampling, especially at higher frequencies, is needed to study in detail the delay between the different wavelengths, the actual time between the ejection of a component and the change in the radio fluxes, and the spectral index in the single dish observations.

Unfortunately we do not have any simultaneous 3 and $7 \mathrm{~mm}$ VLBI observations of the source, but the simultaneous $13 \mathrm{~mm}$ and $7 \mathrm{~mm}$ VLBA observations allowed us to obtain a spectral 

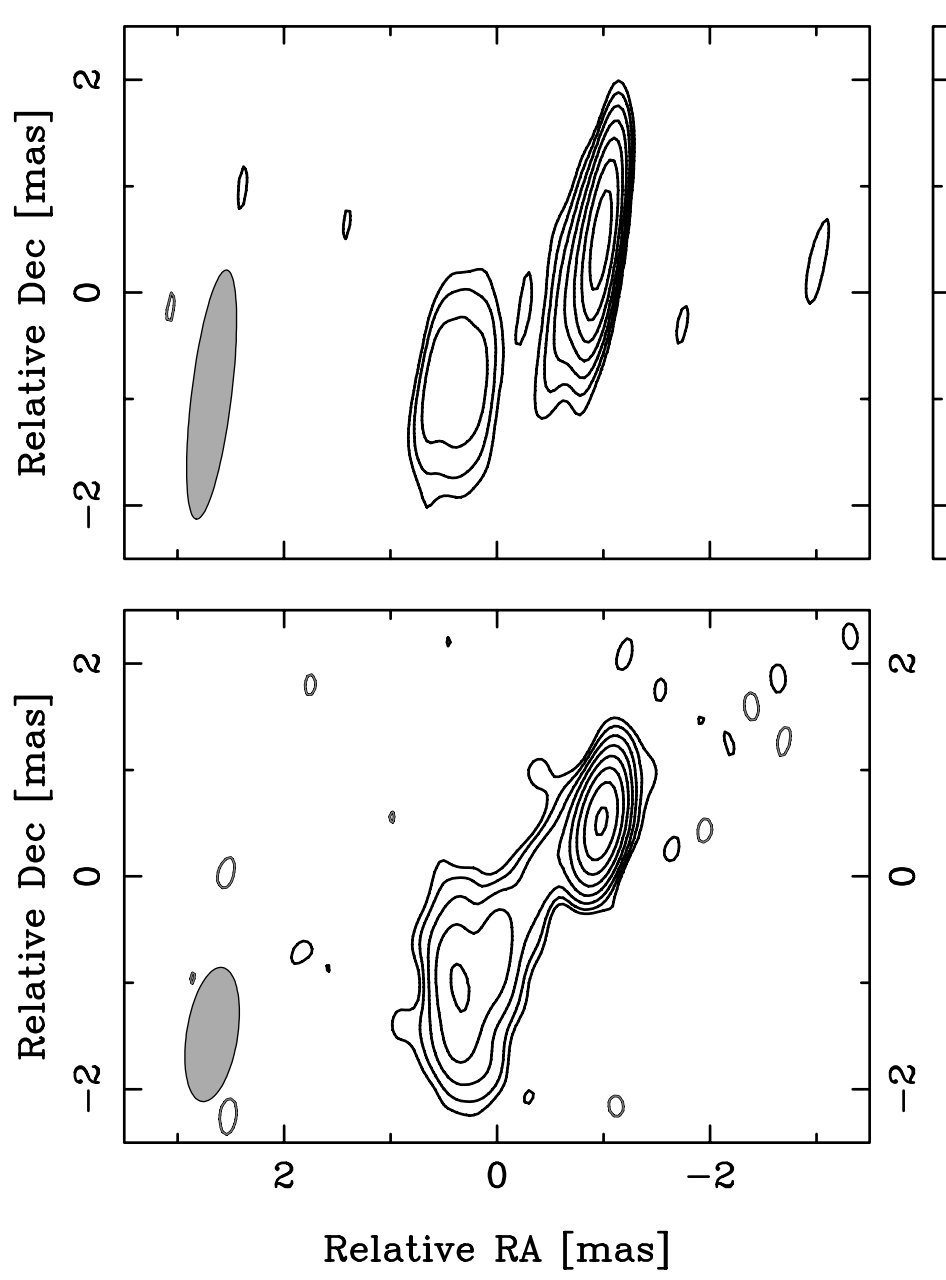

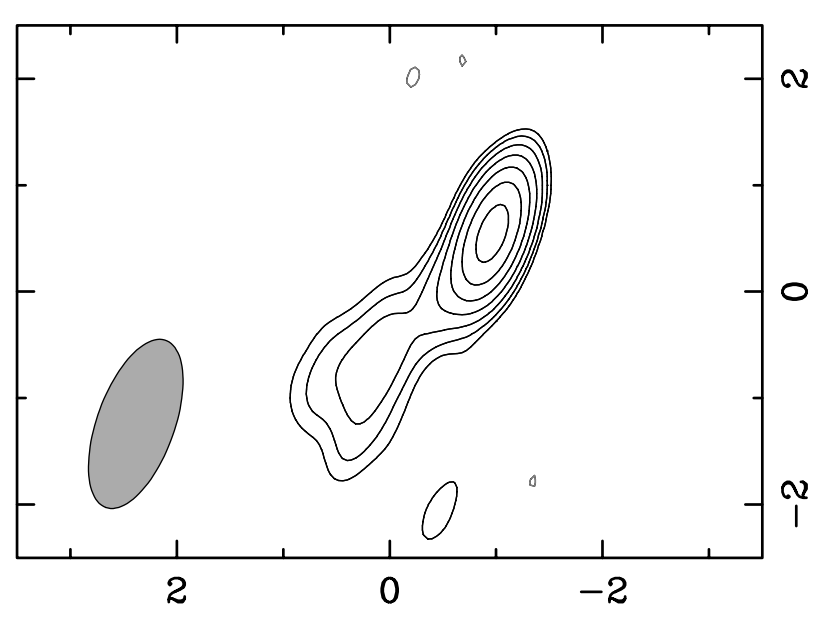

Relative RA [mas]

Fig. 6. CTA $10222 \mathrm{GHz}$ Global VLBI maps. The contour levels in all three maps are $(-3,3,6,12,24, \ldots, 384) \times \mathrm{RMS}$ Noise. Top Left: November 13 1992. Peak Flux $=0.980 \mathrm{Jy} /$ beam and RMS Noise $=3.3 \mathrm{mJy} /$ beam. Convolving beam is an elliptical Gaussian $(1.13 \times 0.19 \mathrm{mas}$ at PA $=-7^{\circ}$ ). Top Right: September 20 1993. Peak Flux $=0.980 \mathrm{Jy} /$ beam and RMS Noise $=4.1 \mathrm{mJy} /$ beam. Convolving beam is an elliptical Gaussian $\left(0.83 \times 0.36\right.$ mas at PA $\left.=-19.8^{\circ}\right)$. Bottom Left: October 191996 . Peak Flux $=0.893 \mathrm{Jy} / \mathrm{beam}$ and RMS Noise $=2.1 \mathrm{mJy} / \mathrm{beam}$. Convolving beam is an elliptical Gaussian $\left(0.64 \times 0.24\right.$ mas at $\left.\mathrm{PA}=-8.8^{\circ}\right)$.

index map of the source (Fig. 11). The spectral index is flat or positive for the core and in the extended component G2. As the dynamic range is insufficient to follow the underlying jet at $43 \mathrm{GHz}$ we cannot determine if the change in the spectral index at $\mathrm{G} 2$ is intrinsic to the source or if it is an artifact caused by the lower dynamic range.

\section{Proper motion of components}

In the following discussion, it is assumed that the observed redshift, $z=1.037$, is cosmological in origin with $H_{\circ}=$ $100 \mathrm{hm} \mathrm{s}^{-1} \mathrm{Mpc}^{-1}$, and $q_{\circ}=0.5$. Using these parameters, 1 mas then corresponds to a distance of $4.2 \mathrm{~h}^{-1} \mathrm{pc}$. At the measured redshift, an angular velocity of $1 \mathrm{mas} \mathrm{yr}^{-1}$ then corresponds to an apparent transverse velocity of $v=28 \mathrm{~h}^{-1} \mathrm{c}$.

\subsection{Distance from the core}

We have calculated the separation from the core individually for each epoch and frequency to avoid problems with the absolute position of the "core", which may differ at different frequencies. We have added the measured distances from the core from Rantakyrö et al. (1996), Wehrle \& Cohen (1989), and Jorstad et al. (2001) to expand the database and to improve the quality of the fits. The errors in the core separation estimates represent the $3 \sigma$ error for the determination of the position of the component. In the case of the observations not determined in this paper, these errors are three times the standard error from the fit to the components (Rantakyrö et al. 1996; Wehrle \& Cohen 1989; Jorstad et al. 2001), while the position measurements presented in this paper are estimated from the spread in solutions in DIFMAPs modelfit software and typically have values of $<15 \%$ of the measured values.

The positions of the components in Jorstad et al. (2001) and in this article agree quite well if error-bars are taken into account. They do all follow a general trend that agrees with the general direction of the jet in this source.

In Fig. 12 the components are plotted as a function of their position in RA and DEC with respect to the core, $D$. The direction of the jet twists almost $90^{\circ}$ at $r \sim 2$ mas, at $r \sim 6$ mas, and, finally at $r \sim 7-8$ mas. The same twisting of the jet is seen directly in Fig. 8. 


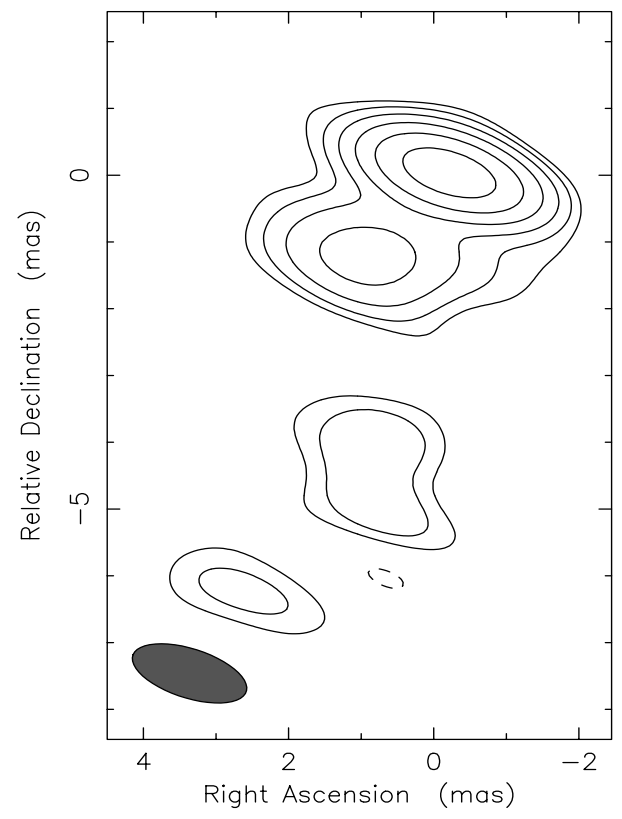

Fig. 7. CTA $10222 \mathrm{GHz}$ map from 1994 March 7. Contour intervals are chosen as $-2,2,4,8,16,32,64$ percent of the peak flux density in the map. The peak flux is $0.39 \mathrm{Jy} /$ beam. The restoring beam is an elliptical Gaussian $\left(1.65 \times 0.73\right.$ mas in $\left.\mathrm{PA}=70^{\circ}\right)$.

\subsection{Proper motion}

In Fig. 13 we have plotted the component separations from the core. Squares indicate distances measured in this paper, those from Rantakyrö et al (1996) and Wehrle \& Cohen (1989) are marked with circles. The diamonds are measurements taken from Jorstad et al. (2001). The vertical fine dotted lines indicate the epochs of observation. The thicker dotted sloped lines show the weighted least squares fit for the proper motion of the components. The fit was done by first fitting the observed $\lambda 3 \mathrm{~mm}$-VLBI observations and then sequentially fitting the components further from the core. We assume that all components are visible within the distance limit of $\leq 10$ mas from the core and they do not disappear and reappear randomly (i.e. the Christmas tree effect causing misidentification). The effect of components disappearing due to sensitivity being too low to detect the weaker components and reappearing again when increasing in apparent flux will not affect our measurements since the components even if undetected are still moving down the jet increasing their distance from the core. Note: Jorstad et al. (2001) had included the 1997.58 B3 component in their fit for motion of B3 and we find a better fit if this is grouped with the $\lambda 3 \mathrm{~mm}$-VLBI observations of the innermost component motion $(\mathrm{J})$. This confusion is understandable due to the limited resolution available with $\lambda 7 \mathrm{~mm}$ VLBA observations. We include a blow-up of the inner 4 mas showing the measured positions of the components and the weighted LSQ fits determining the proper motion in Fig. 14. The symbols used are the same as in Fig. 13.

Table 6 presents the proper motions, $\mu$, in both mas/yr and in $\mathrm{v} / \mathrm{c}$, in the cosmological model that is used (see beginning of Sect. 4), and the zero-epochs calculated from the fitted proper motions. We have labelled the moving components from A to J,
Table 4. Model parameters for the spherical Gaussian components fitted to the CTA 102 maps for the global and EVN sessions 1992, 1993, 1994, and 1996. Flux is the flux density of the component, $\Delta \alpha$ and $\Delta \delta$ gives the position of the component relative to the core, Size is the size of the fitted Gaussian (diameter).

\begin{tabular}{llrrrr}
\hline \hline Epoch & ID & $\begin{array}{r}\Delta \alpha \\
{[\mathrm{mas}]}\end{array}$ & $\begin{array}{r}\Delta \delta \\
{[\mathrm{mas}]}\end{array}$ & $\begin{array}{r}\text { Flux } \\
{[\mathrm{Jy}]}\end{array}$ & $\begin{array}{r}\text { Size } \\
{[\mathrm{mas}]}\end{array}$ \\
\hline 1992 & C0 & 0 & 0 & 0.98 & 0.06 \\
1992 & C1 & 0.19 & -0.28 & 0.17 & 0.18 \\
1992 & C2 & 0.8 & -0.7 & 0.039 & 0.17 \\
1992 & C3 & 1.3 & -1.4 & 0.21 & 0.48 \\
1992 & C4 & 2.8 & -6.64 & 0.40 & 2.8 \\
1993 & D0 & 0 & 0 & 0.53 & $<0.01$ \\
1993 & D1 & 0.03 & -0.08 & 0.54 & $<0.01$ \\
1993 & D2 & 0.45 & -0.56 & 0.091 & 0.084 \\
1993 & D3 & 1.2 & -1.25 & 0.17 & 0.50 \\
1993 & D4 & 1.3 & -2.0 & 0.03 & $<0.01$ \\
1993 & D5 & 1.4 & -4.6 & 0.10 & 2.5 \\
1993 & D6 & 2.8 & -7.0 & 0.20 & 1.6 \\
1993 & D7 & 4.1 & -9.7 & 0.06 & 2.1 \\
1994 & E0 & 0 & 0 & 0.416 & 0.2196 \\
1994 & E1 & 1.0 & -1.2 & 0.14 & 1.2 \\
1994 & E2 & 1.0 & -4.3 & 0.06 & 1.2 \\
1996 & F0 & 0 & 0 & 0.24 & $<0.01$ \\
1996 & F1 & 0.07 & -0.16 & 0.68 & 0.074 \\
1996 & F2 & 0.2 & -0.4 & 0.19 & 0.30 \\
1996 & F3 & 0.6 & -0.7 & 0.04 & $<0.01$ \\
1996 & F4 & 1.2 & -1.4 & 0.34 & 0.64 \\
1996 & F5 & 1.3 & -2.1 & 0.17 & 0.41 \\
1996 & F6 & 1.0 & -4.6 & 0.045 & 0.95 \\
1996 & F7 & 2.9 & -7.0 & 0.34 & 2.86 \\
1996 & F8 & 5.2 & -8.9 & 0.09 & 1.4 \\
\hline & & & & &
\end{tabular}

and this labelling should not be confused with the individual labelling of the components in the maps presented in this paper.

We have determined the proper motion in this source by using all the measured transverse speeds by doing a LSQ fit to the data in Table 6 . The average proper motion is $\sim 0.4 \pm$ 0.07 mas/yr, which corresponds to an apparent transverse velocity of $11 \pm 2 \mathrm{c}$.

The apparent proper motion is a function of the viewing angle, $\theta$, and the component motion in the rest frame of the source, $\beta$ :

$\beta_{\text {app }}=\frac{\beta \sin \theta}{1-\beta \cos \theta}$

The smallest possible $\beta$ can be calculated assuming an optimum $\theta\left(\theta_{\mathrm{opt}}=\arccos \frac{\beta_{\text {app }}}{\sqrt{\beta_{\text {app }}^{2}+1}}\right)$ for the observed proper motion. In the following we will use $\gamma$ when discussing the velocity of the plasma flow. $\gamma_{\min }$ can easily by calculated from $\beta_{\min }$ using:

$\gamma_{\min }=\frac{1}{\sqrt{1-\beta_{\min }^{2}}}$.

Thus for the observed $\beta \sim 11$, we obtain a viewing angle, $\theta \sim$ $5.2^{\circ}$ and the corresponding $\gamma_{\min } \sim 11.1$. 

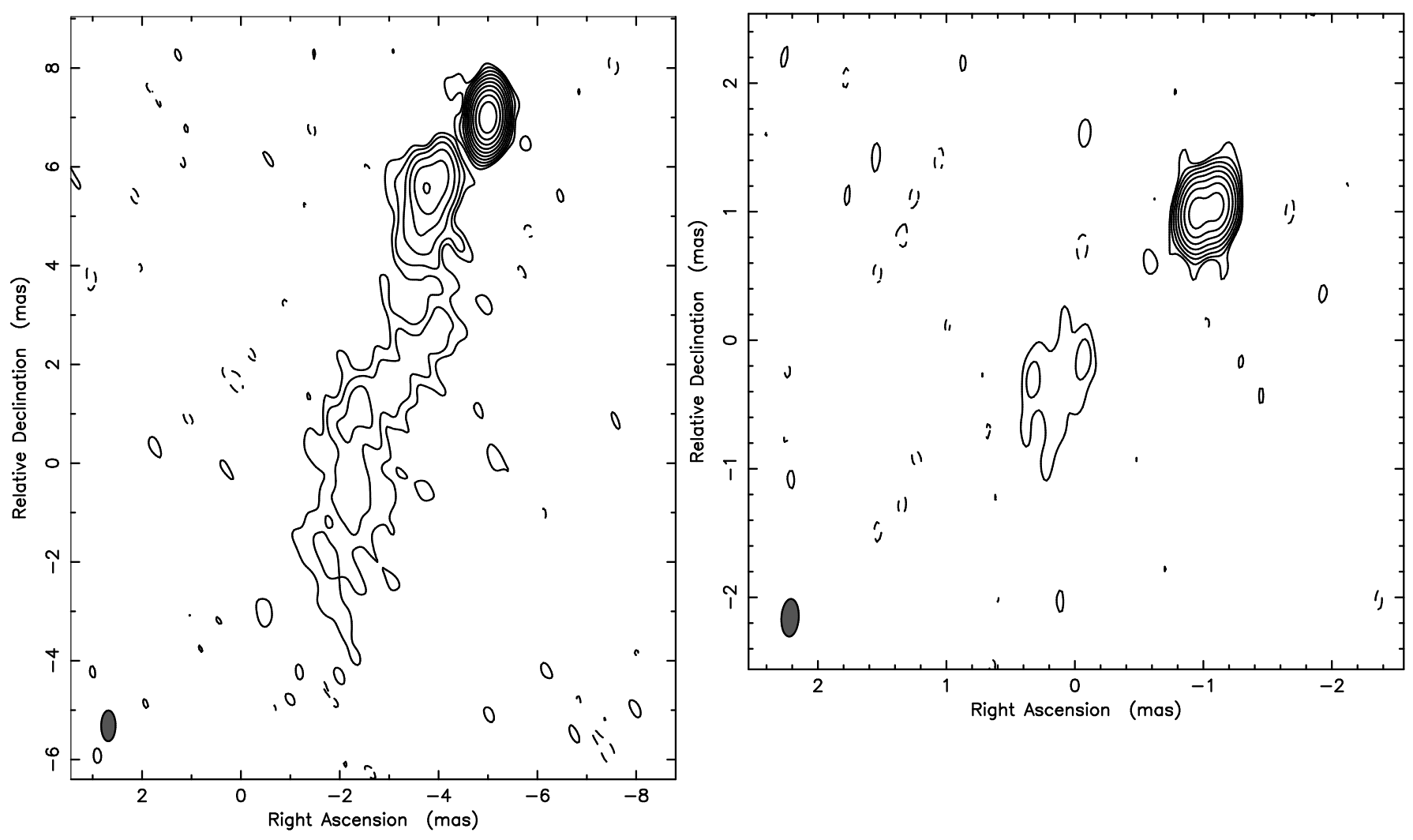

Fig. 8. Left: $22 \mathrm{GHz}$ map from 1998 June 24. Peak flux density $=3.59 \mathrm{Jy} /$ Beam. Contour intervals are chosen as $(-0.1,0.1,0.2,0.4,0.8,1.6$, $3.2,6.4,12.6,25.8,51.2) \times 36 \mathrm{mJy} /$ beam. The restoring beam was a elliptical Gaussian $\left(0.62 \times 0.29 \mathrm{mas}\right.$ in PA $\left.=-0.3^{\circ}\right)$. Right: $43 \mathrm{GHz}$ map from 1998 June 24. Peak flux density $=2.06 \mathrm{Jy} /$ Beam. Contour intervals are chosen as $(-0.5,0.5,1,2,4,8,16,32,64) \times 21 \mathrm{mJy} / \mathrm{beam}$. The restoring beam was a elliptical Gaussian $\left(0.29 \times 0.14\right.$ mas in $\left.\mathrm{PA}=-3.4^{\circ}\right)$.

Table 5. Model parameters for the elliptical Gaussian components fitted to the CTA 102 VLBA maps made in June 24th 1998. $\lambda$ is the observing wavelength in millimeter, ID is the label identifying the component, $\Delta \alpha$ and $\Delta \delta$ give the position of the component relative to the core, Flux is the flux density of the component, $b_{\text {maj }}$ and $b_{\text {min }}$ are the major and minor axes of the elliptical Gaussians $(F W H M)$, and PA is the position angle of the fitted Gaussians.

\begin{tabular}{llrrrrrr}
\hline \hline$\lambda$ & ID & $\begin{array}{r}\Delta \alpha \\
{[\mathrm{mas}]}\end{array}$ & $\begin{array}{r}\Delta \delta \\
{[\mathrm{mas}]}\end{array}$ & $\begin{array}{r}\text { Flux } \\
{[\mathrm{Jy}]}\end{array}$ & $\begin{array}{r}b_{\text {maj }} \\
{[\mathrm{mas}]}\end{array}$ & $\begin{array}{r}b_{\min } \\
{[\mathrm{mas}]}\end{array}$ & $\begin{array}{r}\text { PA } \\
{\left[{ }^{\circ}\right]}\end{array}$ \\
\hline 7 & $\mathrm{G} 0$ & 0 & 0 & 2.1 & 0.08 & 0.04 & -70 \\
7 & $\mathrm{G} 1$ & 0.16 & -0.06 & 2.1 & 0.05 & 0.02 & -87 \\
7 & $\mathrm{G} 2$ & 1.25 & -1.36 & 0.2 & 0.80 & 0.46 & -26 \\
13 & $\mathrm{H} 0$ & 0 & 0 & 1.4 & 0.18 & $<0.01$ & -19 \\
13 & $\mathrm{H} 1$ & 0.14 & -0.03 & 3.0 & 0.16 & 0.07 & -38 \\
13 & $\mathrm{H} 2$ & 1.05 & -1.18 & 0.16 & 0.60 & 0.34 & -4 \\
13 & $\mathrm{H} 3$ & 1.39 & -1.44 & 0.17 & 0.52 & 0.23 & 20 \\
13 & $\mathrm{H} 4$ & 1.38 & -2.14 & 0.13 & 0.94 & 0.66 & -33 \\
13 & $\mathrm{H} 5$ & 2.5 & -6.3 & 0.13 & 5.0 & 1.44 & -22 \\
\hline
\end{tabular}

\section{Component peak brightness temperatures}

With Gaussian components the observed brightness temperature, $T_{\mathrm{B}, \mathrm{obs}}$, is given by:

$T_{\mathrm{B}, \mathrm{obs}}=1.224 \times 10^{12} \frac{S_{v}(1+z)}{v^{2} \times b_{\text {min }} \times b_{\text {maj }}}[\mathrm{K}]$

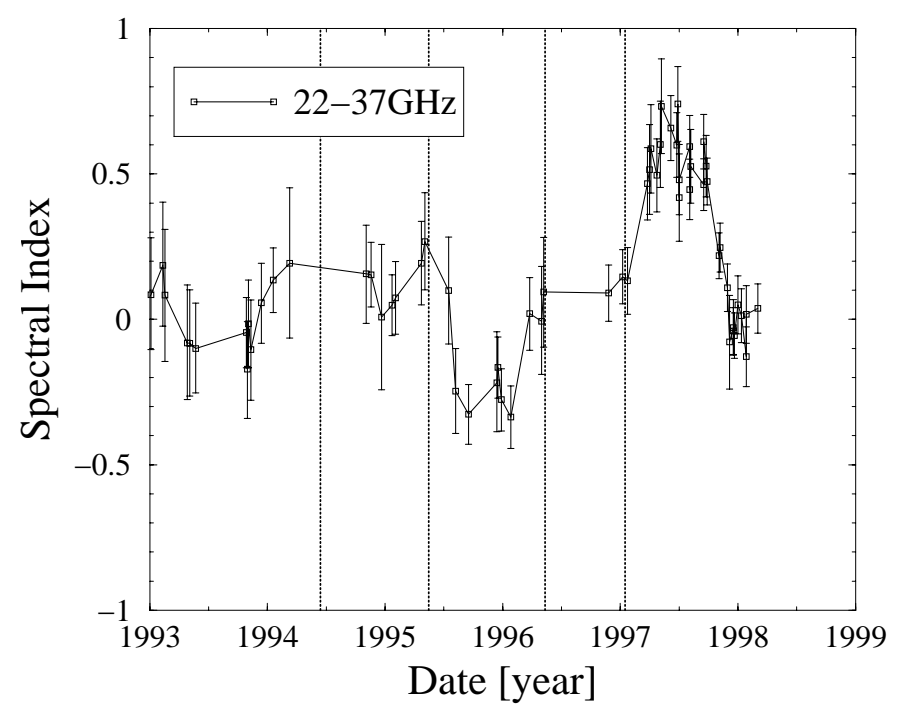

Fig. 9. Spectral index obtained from the 22 and $37 \mathrm{GHz}$ observations made at Metsähovi. The spectral index is defined as $S \propto v^{\alpha}$. The errors bars indicate the $3 \sigma$ limit of the observations. The vertical dotted lines indicate the estimated dates of the zero epochs from the proper motion estimates (see Sect. 4).

where $S_{v}$ [Jy] is the observed peak flux density at the frequency $v[\mathrm{GHz}] . z$ is the redshift, $b_{\text {min }}$ and $b_{\text {maj }}$ are the minor and major axes of the Gaussian component [mas]. Using the 


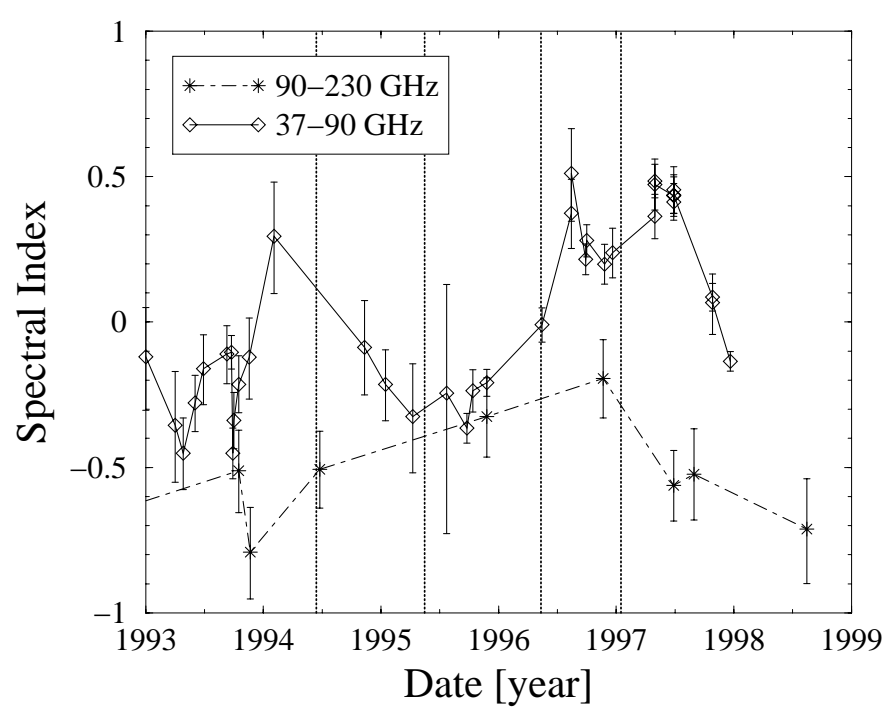

Fig. 10. Spectral index obtained from the 37, 90, and, $230 \mathrm{GHz}$ observations made at Metsähovi and SEST. The spectral index is defined as $S \propto v^{\alpha}$. The errors bars indicate the $3 \sigma$ limit of the observations. The vertical dotted lines indicate the estimated dates of the zero epochs from the proper motion estimates (see Sect. 4).

values in Tables 3-5 and Eq. (3), we obtain the $T_{\mathrm{B} \text {,obs }}$ values presented in Table 7 . Note: $T_{\mathrm{B}, \mathrm{obs} \text {,max }}$ is the brightness temperature obtained by using the Gaussian modelfit parameters and $T_{\mathrm{B} \text {,obs,min }}$ is obtained by using the $1 / 10$ th of dirty beam size if the Gaussian model component size is smaller than this size. Since the $T_{\mathrm{B} \text {,obs }} \mathrm{S}$ are the result of the $T_{\mathrm{B}} \mathrm{S}$ in the source frame multiplied by the Doppler beaming factor, we include the estimated Doppler factor in Table 7. For each component we have calculated the Doppler factor, $D$, using Eq. (4).

$D=\frac{1}{\gamma\left(1-\sqrt{1-1 / \gamma^{2}} \cos \theta\right)}$.

Using the above determined $\theta \sim 5.2^{\circ}$ and the corresponding $\gamma_{\text {min }} \sim 11.1$, the Doppler factor, $D$, is $\sim 11$.

Assuming for a unresolved component close to the core the flux can be expressed as $S \propto S_{0} v^{\alpha}$, and the resolution is inversely proportional to the baseline lengths (NS and EW) and observing frequency, then Eq. (3) can be simplified to:

$T_{\mathrm{B}, \mathrm{obs}} \propto v^{\alpha} \times$ baseline $_{\mathrm{NS}} \times$ baseline $_{\mathrm{EW}}$.

Thus the low values of $T_{\mathrm{B} \text { obs }}$ from the EVN observation are expected because of the short baseline lengths. The $\lambda 3 \mathrm{~mm}$ VLBI observations suffer from smaller baseline lengths compared to Global VLBI, but this is compensated by the positive spectral index of the source in 1997. The most accurate determination of $T_{\mathrm{B} \text {,obs }}$ is from the $22 \mathrm{GHz}$ Global VLBI observations, while the $43 \mathrm{GHz}$ observations are hampered by the spectral index, $\alpha \leq 0$, during the observations. In many cases in the $\lambda 3 \mathrm{~mm}$-VLBI observations the $\chi^{2}$ solutions yielded very thin and narrow elliptical Gaussians. The narrow, thin elliptical Gaussians are the result of the model fitting procedure and are not believed to be a physical representation of the actual shape of the Gaussians. Unfortunately this means that the determined $T_{\mathrm{B} \text {,obs,max }}$ may be highly incorrect, and in these cases

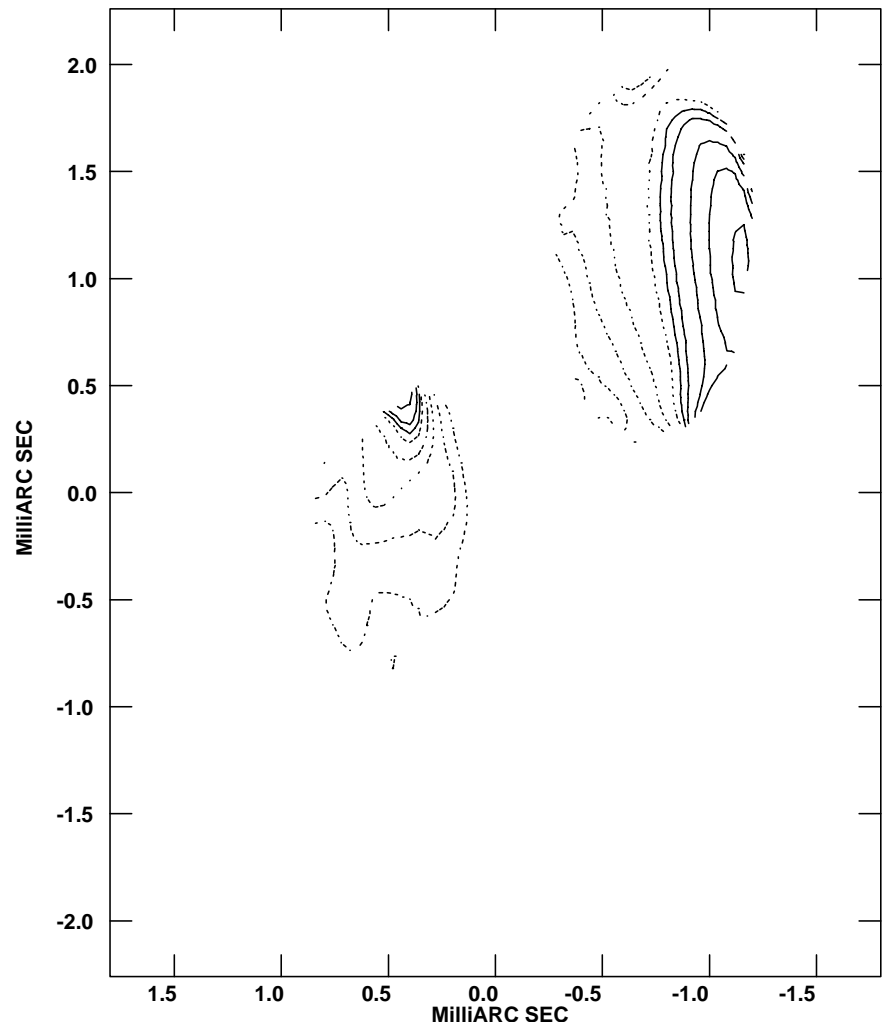

Fig. 11. CTA 102 spectral index map from 1998 June 24, determined using the simultaneous 22 and $43 \mathrm{GHz}$ VLBA maps. Spectral index is defined here as $S \propto v^{\alpha}$. Contour levels are defined as $(-1.5,-1,-0.75,-0.5,-0.3,-0.1,0,0.1,0.3,0.5,0.75,1) \times 1.0$. The first negative contour is shown as a dashed line in the figure.

the $T_{\mathrm{B} \text {,obs,min }}$ may yield a more realistic value. The long thin Gaussians often occur by using the modelling software as it tries to use 1 component when instead 2 or more components makes a better fit. Thus even if these components are extended the software basically fits a thin line connecting these two components underestimating the area of the Gaussians.

Using the logarithmic variability amplitudes and timescales to calculate $T_{\mathrm{B}}$ (Teräsranta \& Valtaoja 1994), we obtain an independent estimate for the brightness temperature. Note: this is the $T_{\mathrm{B}}$ as seen in a reference frame comoving with the emitting component. We later will refer to this reference frame as the "source rest frame". The $T_{\mathrm{B}}$ can be expressed as:

$T_{\mathrm{B}}=5.87 \times 10^{21} \frac{\lambda^{2} \Delta S}{\tau_{\mathrm{obs}}^{2}}\left[1-\frac{1}{\sqrt{1+z}}\right]^{2} \times h^{2}[\mathrm{~K}]$

where $z$ is the redshift, $\lambda$ is the observing wavelength, $\Delta \mathrm{S}$ is the amplitude of the outburst in Jy, $\tau_{\mathrm{obs}}$ is the variability timescale in days, and $h$ is the scaling factor for $H_{\circ}$ (see Sect. 4). $\tau_{\text {obs }}$ is defined as (Burbidge et al. 1974):

$\tau_{\mathrm{obs}}=\frac{\Delta t}{\Delta[\ln (S)]}$.

This method requires a good time-sampling and the $230 \mathrm{GHz}$ data are too sparsely sampled to be suitable for calculations of $T_{\mathrm{B}}$. Applying this to the 1997.0 outburst at 22, 37, and, $90 \mathrm{GHz}$ we obtain: $T_{\mathrm{B}} \sim 5 \pm 2[22 \mathrm{GHz}], 4 \pm 1[37 \mathrm{GHz}]$, and 


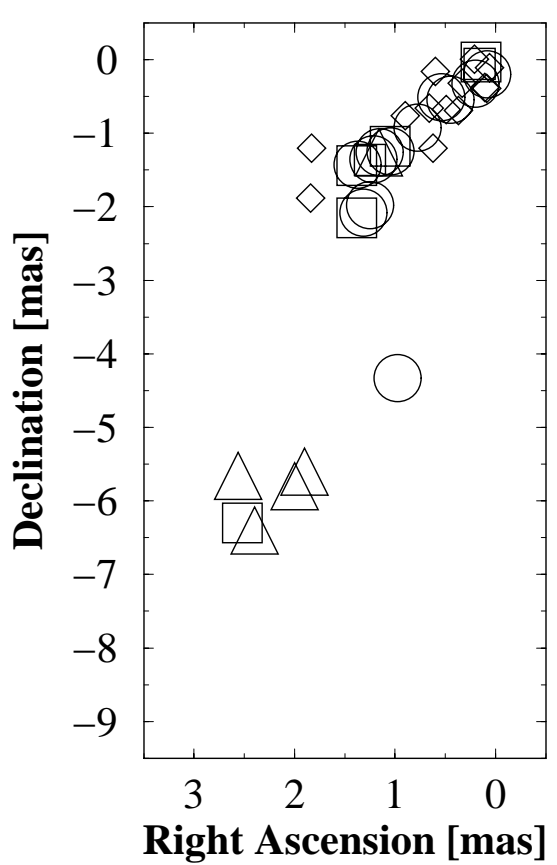

Fig. 12. The position of the fitted Gaussian components are plotted relative to the core, $D$. The observed positions are labelled as, $\diamond, \lambda 3 \mathrm{~mm}$ VLBI, $\bigcirc, 22 \mathrm{GHz}$ observations, $\triangle, 0.9$ to $1.6 \mathrm{GHz}$, and, $\square$, VLBA observations at 22 and $43 \mathrm{GHz}$.

$1 \pm 0.5[90 \mathrm{GHz}] \times 10^{12} \mathrm{~K}$. Even in the best case the available sampling causes us to underestimate the full amplitude of the outburst, and miss the most rapid variations. The critical points in the determination of $T_{\mathrm{B}}$ are the determination of the underlying quiescent flux so that an accurate value of the flux increase due to the outburst can be found, and the confusion caused by superposed flare components. The latter can be avoided to some degree by selecting a suitable outburst that does not exhibit this feature. In determining the quiescent flux we have used the same method as Teräsranta \& Valtaoja (1994), i.e. taking half the flux before the outburst as the quiescent flux. All these factors will underestimate rather than overestimate the $T_{\mathrm{B}} \mathrm{s}$ by as much as a factor of 5 . The sampling and the flux variations are unsuitable to make this calculation for the actual epochs of the $22 \mathrm{GHz}$ observations. Assuming that all outbursts reach similar maximum $T_{\mathrm{B}} \mathrm{S}\left(\sim 1 \times 10^{12}\right)$ and the $\gamma_{\mathrm{S}}(\sim 11)$ determined in Sect. 4.2 describes the relativistic plasma jet, then:

1. The highest measured $T_{\mathrm{B}} \mathrm{s}$ (Sep. 1993, Oct. 1996, and Apr. 1997) all conform with a maximum source frame (i.e. after correcting the observed $T_{\mathrm{B}}$ with the inferred Doppler factor) $T_{\mathrm{B}} \mathrm{S}$ of $\sim$ a few $\times 10^{12} \mathrm{~K}$.

2. The highest values for $T_{\mathrm{B}}$ are all measured in the core or in a component just ejected. This is most clearly seen in $3 \mathrm{~mm}-\mathrm{VLBI}$ observations in 1997 where the highest $T_{\mathrm{B}}$ is obtained in April 1997, just during the rapid increase in the total flux density. The later measurements in 1997 all show significantly lower values for $T_{\mathrm{B}}$.

3. The measured variability of the $T_{\mathrm{B}} \mathrm{S}$ at 22 and $37 \mathrm{GHz}$ in 1997.0 agree well with the $6 \times 10^{12} \mathrm{~K}$ obtained by Teräsranta \& Valtaoja (1994). These values are significantly lower

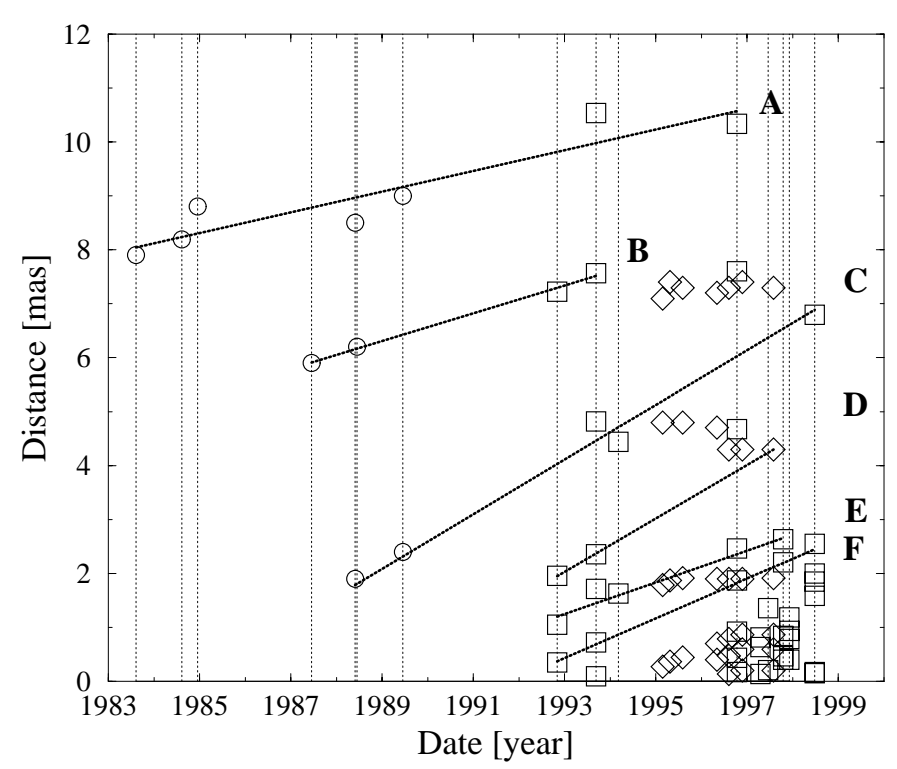

Fig. 13. The position of the fitted Gaussian components are plotted relative to the core, $D$. The lines represent the fitted proper motion to the measured position of the observed components. The moving "components" are labelled from A to I and the proper motion is listed in Table 6. The separations from the core measured in this paper are marked with squares, those from Rantakyrö et al. (1996) and Wehrle \& Cohen (1989) are marked with circles. The diamonds represent the data from Jorstad et al. (2001). The vertical dotted lines indicate the epoches of observations.

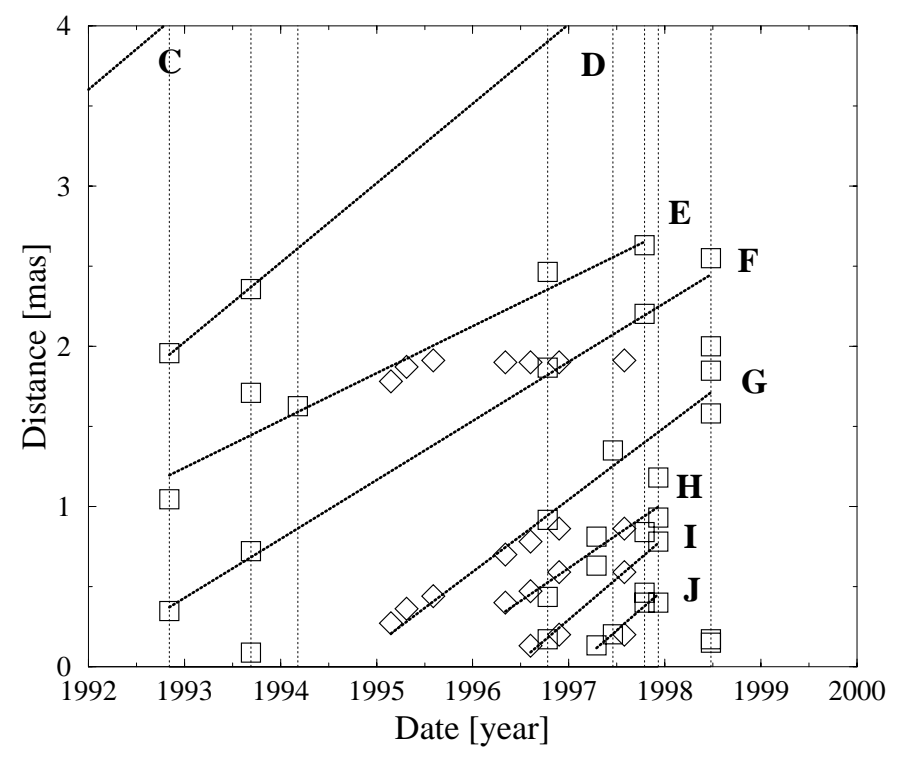

Fig. 14. A blow-up of the inner 4 mas in Fig. 13. Distances from the core measured in this paper are are marked with squares. The diamonds represents the data from Jorstad et al. (2001). The vertical dotted lines indicate the epoches of observations.

than the direct measurements but as discussed previously these measurements are underestimates of the $T_{\mathrm{B}}$.

4. The 1994 measurement yielded very much lower values. This agrees with the flux monitoring which shows the source being in a relative quiescent state after the large outburst in 1992 and before the outburst in 1996. 
Table 6. The proper motions in this Table are obtained from weighted least squares fit to the positions of the components in Fig. 13. The errors are the $3 \sigma$ errors obtained from the fit. The columns are from left to right: ID is the name of the moving component (this label is unique for the proper motion and should not be confused with the labelling used for the individual components in the maps), $\mu$ is proper motion in mas/yr, proper motion in $v / c$, and the zero-epoch when the component was ejected.

\begin{tabular}{lrrr}
\hline \hline ID & $\begin{array}{r}\mu \\
{[\mathrm{mas} / \mathrm{yr}]}\end{array}$ & $v / c$ & $\begin{array}{r}\text { Zero } \\
\text { [year] }\end{array}$ \\
\hline $\mathrm{A}$ & $0.15 \pm 0.04$ & $4.2 \pm 1.1$ & 1930.3 \\
$\mathrm{~B}$ & $0.25 \pm 0.16$ & $7.2 \pm 3.3$ & 1964.4 \\
$\mathrm{C}$ & $0.47 \pm 0.05$ & $13.2 \pm 1.3$ & 1984.4 \\
$\mathrm{D}$ & $0.49 \pm 0.15$ & $13.8 \pm 4.1$ & 1988.9 \\
$\mathrm{E}$ & $0.31 \pm 0.06$ & $8.8 \pm 1.7$ & 1989.2 \\
$\mathrm{~F}$ & $0.37 \pm 0.03$ & $10.4 \pm 0.9$ & 1991.9 \\
$\mathrm{G}$ & $0.39 \pm 0.03$ & $11.0 \pm 0.9$ & 1994.4 \\
$\mathrm{H}$ & $0.37 \pm 0.06$ & $10.3 \pm 1.6$ & 1995.4 \\
$\mathrm{I}$ & $0.43 \pm 0.06$ & $12.0 \pm 1.7$ & 1996.4 \\
$\mathrm{~J}$ & $0.45 \pm 0.07$ & $12.7 \pm 1.9$ & 1997.04 \\
\hline
\end{tabular}

None of the methods yielded measurements of source frame $T_{\mathrm{B}} \mathrm{s}$ significantly greater than $\sim$ a few $\times 10^{12} \mathrm{~K}$. This may be due to the limiting factor described in Eq. (5) and/or intrinsic to the source. We want to state that $3 \mathrm{~mm}$-VLBI is a very useful technique to determine $T_{\mathrm{B}}$ if the observations are done during outbursts when $\alpha>0$.

\section{Conclusions}

This paper represents the several year's work and observations with both single dish telescopes and interferometric arrays, covering wavelengths from $13 \mathrm{~mm}$ to $1.3 \mathrm{~mm}$. Similarly the resolution of the maps covers an order of magnitude from 0.5 mas to $50 \mu$ as. The highest resolution maps are vital to our observations of the rapid structural changes in this source, and thanks to the proper motions determined close to the core (from CMVA observations), we have been able to apply these proper motion measurements to the observations with poorer resolution and a much worse time sampling. Thus we draw the following conclusions:

- The images we have presented clearly demonstrate the rapid structural changes in this source. Using mmVLBI, VLBA at 22 and $43 \mathrm{GHz}$, and Global VLBI we are able to determine the proper motion using component speeds from close to the core out to component speeds determined at a few tenths of mas from the core.

- We find that the observed average proper motion is $\sim 0.4 \pm$ $0.07 \mathrm{mas} / \mathrm{yr}$, which corresponds to an apparent transverse velocity of $11 \pm 2 \mathrm{c}$.

- The multi-band radio flux monitoring shows a source with rapid flux changes and quick changes in the spectral index and the turnover frequency. These changes are connected to the structural changes seen in this source.

- For the stronger outbursts the higher frequencies are leading the lower frequencies. On the other hand at 22 and
Table 7. Brightness temperatures obtained from the Gaussian components fitted to the data. See the running text for a description of the parameters and how they are calculated.

\begin{tabular}{|c|c|c|c|c|c|}
\hline Date & $\begin{array}{r}\text { Freq } \\
{[\mathrm{GHz}]}\end{array}$ & ID & $\begin{array}{l}T_{\mathrm{B}, \mathrm{obs}, \max } \\
{\left[10^{10} \mathrm{~K}\right]}\end{array}$ & $\begin{array}{l}T_{\mathrm{B}, \mathrm{obs}, \min } \\
{\left[10^{10} \mathrm{~K}\right]}\end{array}$ & $\bar{D}$ \\
\hline Nov. 13, 1992 & 22 & $\mathrm{C} 0$ & 170 & 80 & 11 \\
\hline Nov. 13, 1992 & 22 & $\mathrm{C} 1$ & 3 & 3 & 11 \\
\hline Nov. 13, 1992 & 22 & $\mathrm{C} 2$ & 4 & 4 & 11 \\
\hline Nov. 13, 1992 & 22 & $\mathrm{C} 3$ & 0.08 & 0.08 & 11 \\
\hline Nov. 13, 1992 & 22 & $\mathrm{C} 4$ & 0.02 & 0.02 & 11 \\
\hline Sep. 20, 1993 & 22 & D0 & 2700 & 90 & 11 \\
\hline Sep. 20, 1993 & 22 & D1 & 2800 & 90 & 11 \\
\hline Sep. 20, 1993 & 22 & D2 & 7 & 7 & 11 \\
\hline Sep. 20, 1993 & 22 & D3 & 0.4 & 0.4 & 11 \\
\hline Sep. 20, 1993 & 22 & D4 & 15 & 5 & 11 \\
\hline Sep. 20, 1993 & 22 & D5 & 0.01 & 0.01 & 11 \\
\hline Sep. 20, 1993 & 22 & D6 & 0.04 & 0.04 & 11 \\
\hline Sep. 20, 1993 & 22 & D7 & 0.01 & 0.01 & 11 \\
\hline Mar. 7, 1994 & 22 & E0 & 4 & 4 & 11 \\
\hline Mar. 7, 1994 & 22 & E1 & 0.05 & 0.05 & 11 \\
\hline Mar. 7, 1994 & 22 & E2 & 0.02 & 0.02 & 11 \\
\hline Oct. 19,1996 & 22 & F0 & 1200 & 80 & 11 \\
\hline Oct. 19,1996 & 22 & $\mathrm{~F} 1$ & 60 & 60 & 11 \\
\hline Oct. 19,1996 & 22 & $\mathrm{~F} 2$ & 1 & 1 & 11 \\
\hline Oct. 19,1996 & 22 & F3 & 200 & 13 & 11 \\
\hline Oct. 19,1996 & 22 & $\mathrm{~F} 4$ & 0.4 & 0.4 & 11 \\
\hline Oct. 19,1996 & 22 & F5 & 0.5 & 0.5 & 11 \\
\hline Oct. 19,1996 & 22 & F6 & 0.03 & 0.03 & 11 \\
\hline Oct. 19,1996 & 22 & F7 & 0.02 & 0.02 & 11 \\
\hline Oct. 19,1996 & 22 & F8 & 0.02 & 0.02 & 11 \\
\hline Jun. 24, 1998 & 43 & G0 & 90 & 90 & 11 \\
\hline Jun. 24, 1998 & 43 & G1 & 280 & 280 & 11 \\
\hline Jun. 24, 1998 & 43 & $\mathrm{G} 2$ & 0.1 & 0.1 & 11 \\
\hline Jun. 24, 1998 & 22 & $\mathrm{HO}$ & 400 & 140 & 11 \\
\hline Jun. 24, 1998 & 22 & $\mathrm{H} 1$ & 140 & 140 & 11 \\
\hline Jun. 24, 1998 & 22 & $\mathrm{H} 2$ & 0.4 & 0.4 & 11 \\
\hline Jun. 24, 1998 & 22 & H3 & 0.7 & 0.7 & 11 \\
\hline Jun. 24, 1998 & 22 & $\mathrm{H} 4$ & 0.1 & 0.1 & 11 \\
\hline Jun. 24, 1998 & 22 & H5 & 0.004 & 0.004 & 11 \\
\hline Apr. 14, 1997 & 86 & I0 & 1700 & 300 & 11 \\
\hline Apr. 14, 1997 & 86 & I1 & 2.4 & 0.4 & 11 \\
\hline Apr. 14, 1997 & 86 & $\mathrm{I} 2$ & 20 & 4 & 11 \\
\hline Apr. 14, 1997 & 86 & I3 & 10 & 2 & 11 \\
\hline Jun. 18, 1997 & 86 & $\mathrm{~J} 0$ & 5 & 5 & 11 \\
\hline Jun. 18, 1997 & 86 & $\mathrm{~J} 1$ & 140 & 30 & 11 \\
\hline Jun. 18, 1997 & 86 & $\mathrm{~J} 2$ & 80 & 16 & 11 \\
\hline Oct. 24,1997 & 86 & K0 & 40 & 2 & 11 \\
\hline Oct. 24, 1997 & 86 & K1 & 130 & 8 & 11 \\
\hline Oct. 24,1997 & 86 & K2 & 260 & 15 & 11 \\
\hline Oct. 24, 1997 & 86 & K3 & 100 & 6 & 11 \\
\hline Oct. 24,1997 & 86 & K4 & 50 & 3 & 11 \\
\hline Oct. 24,1997 & 86 & K5 & 50 & 3 & 11 \\
\hline Dec. 13, 1997 & 86 & L0 & 340 & 84 & 11 \\
\hline Dec. 13,1997 & 86 & L1 & 4 & 4 & 11 \\
\hline Dec. 13, 1997 & 86 & L2 & 50 & 10 & 11 \\
\hline Dec. 13,1997 & 86 & L3 & 20 & 4 & 11 \\
\hline Dec. 13, 1997 & 86 & L4 & 10 & 3 & 11 \\
\hline
\end{tabular}

$37 \mathrm{GHz}$ the outbursts are almost simultaneous in time as is expected by the shock in jet model. 
- The determination of the proper motions show a highly relativistic jet, and allows us to extrapolate backwards to find the zero epochs of the outbursts. We see that at the start of each large outburst there is an ejection of a component from the core. Thus the total flux monitoring is a valuable tool in helping us understand the structural changes in this source. The ejection of a component from the core is also seen in the spectral index plots, where each ejection causes a steepening in the spectrum. Better timesampling, especially at higher frequencies, is needed to study the delay between the bands in detail, as well as the actual time between an ejection of a component and the change in the radio fluxes and spectral indexes in the single dish observations.

- The determination of the source frame $T_{\mathrm{B}}$ of the individual components agree both with the current models for the ejection of components in relativistic plasma jets and estimates of source frame $T_{\mathrm{B}} \mathrm{S}$ using the logarithmic variability amplitudes and timescales. These observations also show a connection between the outbursts in the total flux monitoring and the high source frame $T_{\mathrm{B}} \mathrm{s}$ determined from the interferometer images.

We conclude that further studies of this source are needed, including high resolution images and observations at longer wavelengths in order to determine the spectral indexes as a function of time and of distance from the core. The single dish monitoring has been of great value and should be a fundamental part of future observations of this source. Most important is that the observations should be done with short times between the sessions to obtain the smallest possible error in the determination of the proper motion of each component. With an observed proper motion of $\sim 0.4 \pm 0.07 \mathrm{mas} / \mathrm{yr}$ we would ideally need a maximum of 3 months between observations.

Acknowledgements. We would like to thank the people at the individual telescopes for helping in making the observations. Without their support these observations would not have been possible. We would also like to thank the people at the Haystack who are organizing the CMVA. The SEST is operated by ESO and the Swedish National Facility for Radio Astronomy, Onsala Space Observatory at Chalmers University of Technology. Onsala Space Observatory at Chalmers University of Technology is the Swedish National Facility for Radio Astronomy. The Hat Creek array is operated by the Berkeley-IllinoisMaryland Association with funding from the National Science Foundation, Grant AST 93-20238 to the University of California. The NRAO is a facility of the National Science foundation, operated under cooperative agreement by Associated Universities, Incorporated. FCRAO is operated with support of the National Science Foundation and with permission of the Metropolitan District Commission of the Commonwealth of Massachusetts. This is contribution number 819 of the Five College Astronomy Department. The Haystack Observatory is operated by the Massachusetts Institute of Technology, with support from the NSF, NASA, and the USAF. F. T. Rantakyrö gratefully acknowledges support from the Chilean Centro de Astrofísica FONDAP
No. 15010003. M.T. acknowledges the support of the Academy of Finland for the Finnish AGN flux density monitoring projects with the SEST. K.W. acknowledges grants from Emil Aaltonen Foundation and Vilho, Yrjö and Kalle Väisälä Foundation for part of this work. We would like to thank Dr. Jeff Kenney for proof reading this manuscript. This research has made use of the NASA/IPAC Extragalactic database (NED), which is operated by the Jet Propulsion Laboratory, Caltech, under contract with the National Aeronautics and Space Administration. This research has also made use of NASA's Astrophysics Data System Abstract Service (ADS).

\section{References}

Alberdi, A., Gómez, J. L., Marcaide, J. M., Marscher, A. P., \& Pérez-Torres, M. A. 2000, A\&A, 361, 529

Blandford, R. D., \& Königl, A. 1979, ApJ, 232, 34

Burbidge, G., Jones, T. W., \& O’Dell, S. L. 1974, ApJ, 193, 43

Bååth, L. B., Rogers, A. E. E., Inoue, M., et al. 1992, A\&A, 257, 31

Courvoisier, T. J. -L., Robson, E. I., Hughes, D. H., Blecha, A., \& Bouchet, P. 1988, Nature, 335, 330

Dent, W. A. 1965, AJ, 70, 672

Jorstad, S., Marscher, A. P., Mattox, J. R., et al. 2001, ApJS, 134, 181

Lainela, et al. 1993, in Variability in Blazars, ed. E. Valtaoja, \& M. Valtonen (Cambridge: Cambridge University Press), 102

Litchfield, S. J., Stevens, J. A., Robson, E. I., \& Gear, W. K. 1995, MNRAS, 274, 221

Marscher, A. P., \& Gear, W. 1985, ApJ, 421, 153

Marscher, A. P., Gear, \& Travis W. 1992, in Variability in Blazars, ed. E. Valtaoja, \& M. Valtonen (Cambridge: Cambridge: University Press), 85

Nolan, P. L., Bertsch, D. L., Fichtel, C. E., et al. 1993, ApJ, 414, 82

Rantakyrö, F. T., Bååth, L. B., \& Matveenko, L. 1995, A\&A, 293, 44

Rantakyrö, F. T., Bååth, L. B., Dallacasa, D., Jones, D. L., \& Wehrle, A. E. 1996, A\&A, 310, 66

Rantakyrö, F. T., Bååth, L. B., Backer, D. C., et al. 1998, A\&AS, 131, 451

Rickett, B. J., Coles, W. A., \& Bourgois, G. 1984, A\&A, 134, 390

Rickett, B. J. 1986, ApJ, 307, 564

Romney, J., Padrielli, L., Bartel, N., et al. 1984, A\&A, 134, 390

Scheuer, P. A. G., \& Readhead, A. C. S. 1979, Nature, 277, 182

Scholomitski, G. B. 1965, AZh, 9, 3, 516

Shepherd M. C. 1997, ASP Conf. Ser., 125, Astronomical Data Analysis Software and Systems VI, 6, 77

Slish, V. I. 1963, Nature, 199, 682

Spencer, R. E., McDowell, J. C., Charlesworth, M., et al. 1989, MNRAS, 240, 647

Stevens, J. A., Litchfield, S. J., Robson, E. I., et al. 1996, ApJ, 466, 158

Teräsranta, H., \& Valtaoja, E. 1994, A\&A, 283, 51

Teräsranta, H., Tornikoski, M., Mujunen, A., et al. 1998, A\&AS, 132, 305

Tornikoski, M., Valtaoja, E., Teraesranta, H., et al. 1996, A\&AS, 116, 157

Tornikoski, M., et al. 2002, A\&A, in preparation

Wehrle, A. E., \& Cohen, M. H. 1989, ApJ, 346, L69

Wiik, K., Valtaoja, E., \& Leppänen, K. 2001, A\&A, 380, 72 